\title{
Quality of Energy Provisioning for Wireless Power Transfer
}

\author{
Haipeng Dai, Member, IEEE, Guihai Chen, Member, IEEE, Chonggang Wang, Member, IEEE, \\ Shaowei Wang, Member, IEEE, Xiaobing Wu, Member, IEEE, and Fan Wu, Member, IEEE
}

\begin{abstract}
One fundamental question for wireless power transfer technology is the energy provisioning problem, i.e., how to provide sufficient energy to mobile rechargeable nodes for their continuous operation. Most existing works overlooked the impacts of node speed and battery capacity. However, we find that if the constraints of node speed and battery capacity are considered, the continuous operation of nodes may never be guaranteed, which invalidates the traditional energy provisioning concept. In this paper, we propose a novel metric - Quality of Energy Provisioning (QoEP) - to characterize the expected portion of time that a node sustains normal operation by taking into account node speed and battery capacity. To avoid confining the analysis to a specific mobility model, we study spatial distribution instead. As there exist more than one mobility models corresponding to the same spatial distribution, and different mobility models typically lead to different QoEPs, we investigate upper and lower bounds of QoEP in 1D and 2D cases. We derive tight upper and lower bounds of QoEP for 1D case with single source, and tight lower bounds and loose upper bounds for general 1D and 2D cases with multiple sources. Finally, we perform extensive simulations to verify our theoretical findings.
\end{abstract}

Index Terms-Quality of energy provisioning, wireless power transfer, mobility.

\section{INTRODUCTION}

$\mathrm{T}$ HE past few decades witnessed the major success of wireless communication networks and pervasion of wireless and portable mobile devices. Among hot issues like capacity, robustness in wireless communication, conserving energy is particularly important because mobile devices are typically powered by batteries with limited energy. When batteries are used up, they need to be replaced or charged by a wired power plug, which is laborious and sometimes impossible (such as charging batteries of implanted devices). This problem can be overcome by recent advances in wireless power transfer technology [1], which allows energy to be transferred from one storage device to another wirelessly with reasonable efficiency. Without the hassle of wires, this promising technology revolutionizes the way energy is transferred and has been applied to recharge mobile devices such as sensors [2], RFIDs [3], laptops [4], cell phones [5], vehicles [6] and unmanned planes [7]. According to a recent report, the wireless power transfer market is expected to grow to US $\$ 23.7$ billions in 2015 [8].

Generally, the purpose of adopting wireless power

- H. Dai, G. Chen and X. Wu are with the State Key Laboratory for Novel Software Technology, Nanjing University, China, Nanjing 210023.

E-mail: dhpphd2003@gmail.com, \{gchen,wuxb\}@nju.edu.cn.

- C. Wang is with the InterDigital Communications, PA 19406 E-mail: cgwang@ieee.org.

- S. Wang is with the School of Electronic Science and Engineering, Nanjing University, Nanjing 210023, China.

E-mail: wangsw@nju.edu.cn.

- F. Wu is with the Department of Computer Science and Engineering, Shanghai Jiao Tong University, Shanghai 200240, China.

E-mail: fwu@cs.sjtu.edu.cn. transfer technology in typical applications is to guarantee the working performance of mobile devices (we call nodes hereafter for abstraction). Since energy sources (or called wireless chargers in some literature) are expensive, it is not cost-effective to deploy a dense network of stationary sources to provide sufficient power to mobile nodes at every location in the interested area. Instead, as pointed out by He et al. [9], we can exploit mobility of nodes to reduce the number of required sources. This is mainly based on the intuition that a mobile node can harvest and store superfluous energy in its battery within power-rich areas, and then use the energy to sustain its normal working in power-deficient areas. In other words, mobility nature of nodes allows the existence of power-deficient areas and thus leaves room for possible optimization of deployment of sources. The goal of work in [9] is to make nodes energy provisioned, i.e., to guarantee nodes harvesting enough energy for continuous operation, while minimizing the number of sources. This is also termed the energy provisioning problem.

As a pioneer work studying the energy provisioning problem, He's research [9] is mainly confined to triangular deployment and omnidirectional charging model. In addition, it simply requires that the accumulated recharged energy during the node's movement should be no smaller than the total energy consumption of the node in the long run, which completely overlooks the impacts of node speed and battery capacity. We relax restrictions on deployment and charging model in this paper. We further show that after considering the constraints of node speed and battery capacity, the continuous operation of a node may never be guaranteed, which invalidates the energy provisioning concept. 


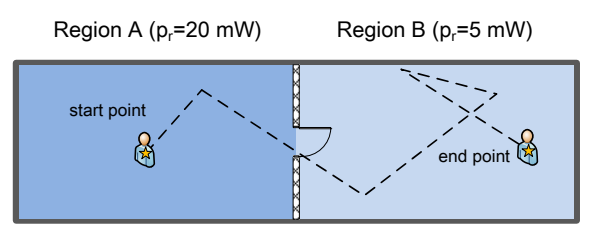

(a) Illustration of the man's movement

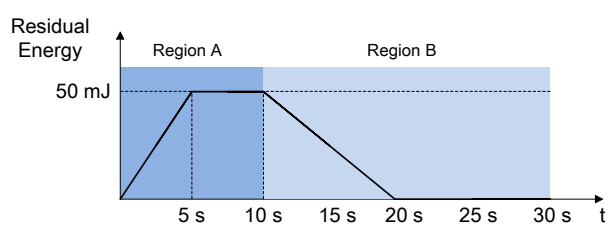

(b) Battery residual energy vs. Time $(v=1 \mathrm{~m} / \mathrm{s})$

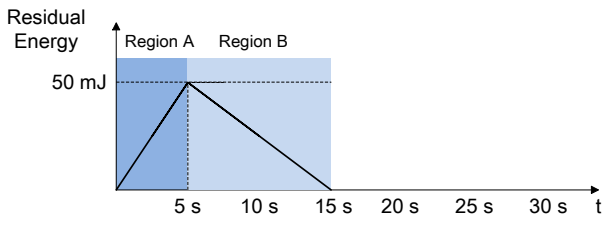

(c) Battery residual energy vs. Time $(v=2 \mathrm{~m} / \mathrm{s})$

Fig. 1: An example showing how node speed affects the Quality of Energy Provisioning. The lengths of trajectory in Region A and Region B are $10 \mathrm{~m}$ (meter) and $20 \mathrm{~m}$, respectively. The tag has battery capacity $50 \mathrm{~mJ}$, and its working power is $10 \mathrm{~mW}$.

We use a simple example to illustrate this issue. We consider a tag worn by a man with working power $10 \mathrm{~mW}$ and battery capacity $50 \mathrm{~mJ}$ (note that all parameter values in this example are arbitrarily calibrated). The man first moves within a power-rich area, Region A as shown in Fig. 1(a), for $10 \mathrm{~m}$ (meter) with constant charging power $20 \mathrm{~mW}$ (typically, charging power is not uniform across space. We use the uniform assumption here to simplify the analysis), and then transfers to a powerdeficient area Region B with constant charging power $5 \mathrm{~mW}$, and continues to move within it for $20 \mathrm{~m}$. Suppose the moving speed $v$ of the man is constant and is equal to $1 \mathrm{~m} / \mathrm{s}$. Obviously, the tag is energy provisioned as its cumulative charging energy $(20 m W * 10 s+5 m W * 20 s=$ $300 \mathrm{~mJ}$ ) is exactly equal to the total required energy for normal working $(10 \mathrm{~mW} * 30 \mathrm{~s}=300 \mathrm{~mJ})$.

Notwithstanding, the amount of energy the tag can store is only $50 \mathrm{~mJ}$. We plot the relation between the battery energy status of tag and time in Fig. 1(b). It can be observed that up to $50 \mathrm{~mJ}$ is wasted at the time interval $\left[\begin{array}{lll}5 & s & 10\end{array}\right]$. As a result, after leaving Region A, the tag depletes all its stored energy and forced to switch off when $t=20 s$ in Region $\mathrm{B}$, and works in an intermittent working mode until $t=30 \mathrm{~s}$. However, if we double the speed of man to $v=2 \mathrm{~m} / \mathrm{s}$, which implies the dwelling time of the tag in each region will be cut in half, the recharge energy loss in Region A can be avoided, as Fig. 1(c) illustrates. The tag can thus keep working during the time period $\left[\begin{array}{lll}0 & s & 15\end{array}\right]$. Likewise, we can image that if the battery capacity of the tag is enlarged to $100 \mathrm{~mJ}$, the tag can keep working all the time, when the moving speed is $1 \mathrm{~m} / \mathrm{s}$.
In fact, given the constraints of battery capacity and node speed, it is always possible for a mobile node obeying some mobility model with stochastic properties, such as Random Waypoint Mobility Model [10], to deplete its energy and suspend work whenever it happens to linger too long in power-deficient areas with charging power being less than the node's working power. Now that continuous working of a node can never be guaranteed under the existence of power-deficient areas, the rigorous energy provisioning becomes invalid.

To evaluate the performance of energy provisioning for mobile nodes when continuous operation of nodes cannot necessarily be guaranteed, we define Quality of Energy Provisioning (QoEP) as the expected portion of time that a node can sustain normal operation in the long run. It captures the characteristics of energy provisioning performance even when the node works in an intermittent mode. As it reveals the long term working performance of the node itself, QoEP is essential for many monitoring applications. For example, the health monitoring devices carried by the potential or high-risk patients and powered by deployed wireless chargers [11] are expected to work as much as possible, which calls for a high QoEP. In general, QoEP serves as a useful metric and can be incorporated into many system designs.

In this paper, we prefer to investigate QoEP of a node based on its spatial distribution, i.e., with what probability the node will stay at a specific location, rather than a specific mobility model in that the former is more general, practical and tractable. Accordingly, our work turns to pursuing upper and lower bounds of QoEP given the spatial distribution, as there exist multiple mobility models with different QoEPs obeying the same spatial distribution. Theoretical results provide insights to many applications, e.g., deploying energy sources to meet requirements for energy provisioning performance, striking a good tradeoff between sensing quality and monitoring time (note that lower sensing rate will lead to lower sensing quality and lower working power, and thus longer monitoring time), and even guiding the movements of nodes.

We make the following main contributions.

- We are the first to study the quality of energy provisioning for mobile nodes given a network of stationary sources. We disclose the impacts of node speed and battery capacity on energy provisioning and present a new metric - Quality of Energy Provisioning (QoEP). Our work is done in such a manner that all its results are applicable to general mobility models, deployment of sources and charging models.

- We obtain tight upper and lower bounds for 1D case with a single source. In particular, we propose a novel analytical approach - flow pattern analysis - by drawing an analogy to flow in physics to facilitate our analysis. This approach not only enables us to design the optimal mobility model, which achieves the maximum QoEP, but also allows 
an effective algorithm to calculate the tight upper bound.

- We further extend the results to general $1 \mathrm{D}$ and 2D cases with multiple sources, and derive tight lower bounds and loose upper bounds in both cases.

- We conduct extensive simulations to verify our theoretical findings. Simulation results show that our upper and lower bounds perfectly hold, and are more practical than existing works.

Our prior work has aimed to derive the upper and lower bounds for the 1D case with single/multiple sources for wireless rechargeable sensor networks [12]. In contrast, we consider the problem in more general scenarios using wireless power transfer technology, and obtain theoretical results for the 2D case with multiple sources as well as useful insights into the 3D case.

The rest of the paper is organized as follows. In Section 2 , we review the related work. Section 3 presents the problem definition and related conceptions. Section 4 contains our results including tight upper and lower bounds for 1D case with single source. We extend our results to 1D and 2D cases with multiple sources in Section 5 and 6, respectively. Further discussions is presented in Section 7. In Section 8, we give the simulation results to support our theoretical findings. Finally, we conclude this work in Section 9.

\section{Related Work}

In this section, we briefly review the related work about wireless power transfer technology and energy provisioning problem.

In recent years, Intel developed the wireless identification and sensing platform (WISP) by integrating RFID tags with sensing and computing components [13]. The RFID tags can be wirelessly charged by readers. Buettner et al. explored this technology to recognize human activities [14]. Exploiting wireless power transfer technology to replenish sensor nodes has also been studied for years. In [15], Powercast developed a wireless power platform to work with wireless sensor network in order to help monitor temperature and humidity at a zoo without disrupting the animal exhibit. In [16], Wicaksono et al. studied the problem of how to deploy stationary energy sources and allocate frequency bands considering the power interference to charge sensor nodes in buildings. Different from the works solely considering the charging efficiency, Dai et al. [17] paid attention to controlling the detrimental effect of electromagnetic radiation.

In view of the expensive cost of energy sources, much recent literature proposed to mobilize single or multiple energy sources to recharge sensor nodes. [18] and [19] studied the problem of how to jointly mobile chargers and schedule sensor nodes to enhance the data routing performance of the entire sensor network. [20] and [21] employed a mobile charger to improve the data gathering efficiency. In [22] and [23], a mobile charger was used not only as an energy transporter, but also as a data collector. Besides, Xie et al. [24] investigated the problem of co-locating the mobile base station on the wireless charging vehicle to minimize energy consumption of the network while ensuring none of the sensor nodes depletes its energy. Still other works concentrated on stochastic event capture issues. Dai et al. [25] considered the problem of selecting sensors for charging and scheduling the sensors'activation schedules to maximize the overall quality of monitoring. Most recently, scientists focused on utilizing multiple mobile energy sources to charge a large-scale sensor network considering their energy constraint. Zhang et al. [26] aimed to maximize the energy efficiency of charging, while Dai et al. [27] attempted to minimize the number of required mobile chargers to guarantee continuous operation of all nodes.

For energy provisioning problem, $\mathrm{He}$ et al. [9] proposed the first scheme to the energy provisioning problem. In [11], Chiu et al. exploited the mobility of enddevices to deploy wireless chargers with partial coverage, with an objective to maximize the survival rate of end-devices.

\section{Problem Statement}

\subsection{Network Model, Mobility Model and Energy Con- sumption Model}

Suppose there is a network of sources and a mobile node (e.g., it is worn by a human user for activity monitoring) in a region of interest. Sources are responsible for recharging the node via wireless. The node harvest wireless energy and store it in its battery for normal operations like sensing and logging data. Moreover, the data reporting process is supposed to be delay-tolerant and occur in a batch manner, thus can be ignored. This wireless recharge infrastructure is generic and can be reused for diverse types of different applications such as WISP [13] and Powercast wireless power platform [15].

Suppose that the node is attached to a mobile object, and thus its movement is largely determined by the object and dictated by a mobility model. In addition, suppose the speed of the node is no more than $v^{\max }$. The node's power consumption for working, such as sensing and logging data, is constant and independent of its motion, which we denote as $p_{s}$. Note that the node spends no energy on movement as it is driven by the object that it is attached to.

The node has a battery capacity of $E_{\pi}$, and its power leakage of battery is negligible. Moreover, it keeps working with a nonzero residual energy until depletion, and then it suspends its work. As long as the node absorbs any amount of energy, it resumes work immediately. The energy cost for switching on or off is typically much smaller than the working energy cost, and therefore is ignored in this paper. Though the last assumption is somewhat unrealistic, it makes the problem much easier to solve. Besides, we can fill the gap between this assumption and the reality by designing more intelligent switching policies (such as setting an energy threshold 
TABLE 1: Notations

\begin{tabular}{|c|c|}
\hline Symbol & Definition \\
\hline$v^{\max }$ & The maximum speed of the node. \\
\hline$p_{s}$ & $\begin{array}{l}\text { The constant nodal power consumption for } \\
\text { working. }\end{array}$ \\
\hline$E_{\pi}$ & The battery capacity of the node. \\
\hline$p_{r}(t)\left(p_{r}(x)\right)$ & $\begin{array}{l}\text { The cumulative charging power node receives } \\
\text { at time } t \text { (location } x \text { ). }\end{array}$ \\
\hline$E_{r e}(t)\left(E_{r e}(x)\right)$ & The residual energy at time $t$ (location $x$ ). \\
\hline$f_{d i s}(x)$ & The spatial distribution at location $\mathrm{x}$. \\
\hline$\lambda_{c}(x)\left(\Lambda_{c}\left(\Omega^{\prime}\right)\right)$ & $\begin{array}{l}\text { The expected battery energy consumption rate } \\
\text { for location } x \text { (subregion } \Omega^{\prime} \text { ). }\end{array}$ \\
\hline$\lambda_{h}(x)\left(\Lambda_{h}\left(\Omega^{\prime}\right)\right)$ & $\begin{array}{l}\text { The expected battery energy harvest rate for } \\
\left.\text { location } x \text { (subregion } \Omega^{\prime}\right) \text {. }\end{array}$ \\
\hline$p_{e p}(x)$ & The energy providing ability for location $x$. \\
\hline
\end{tabular}

and letting the node to switch on if and only if the stored energy level exceeds the threshold) to avoid frequent switching operations and thereby save energy. We will take into account practical concerns in future work.

In some applications, nodes are scheduled to sense and $\log$ date for some time, and sleep regularly to save energy in a cycled fashion. We then treat the average energy power $\overline{p_{s}}$ in one schedule period as $p_{s}$.

For clarity of presentation, we list the main notations used in this paper in Table 1.

\subsection{Charging Model}

The typical charging model adopted in most prior work is proposed by [9]. This empirical model assumes that the charging power of sources is omnidirectional, i.e., any node with the same distance to the source receives the same amount of power. This assumption, however, is not applicable for sources with directional antennas, such as TX91501 power transmitters produced by Powercast [2] with $60^{\circ}$ beam pattern. In this paper, we basically make no specific assumptions with respect to recharging model (except for the 1D case with single source, in which we make an assumption to simplify the analysis), and therefore, our work is general enough to accommodate different recharging models. After all, we assume that the charging power distribution $p_{r}(x)$ has already been determined by some charging models or even statistics of charging power data.

\subsection{Concept of Quality of Energy Provisioning}

As we mentioned before, the sustainable operation of a node can never be achieved whenever there exist power-deficient areas with insufficient charging power to support the node's normal working, thus it is imperative for us to propose a new metric to evaluate the performance of energy provisioning in this case. Before formally stating our metric, we introduce the following definitions in advance.

Definition 3.1: Instantaneous Quality of Energy Provisioning (IQoEP) of a node at time $t$ is defined as follows:

$$
\operatorname{IQoEP}(t)= \begin{cases}\frac{p_{r}(t)}{p_{s}}, & E_{r e}(t)=0 \text { and } p_{r}(t)<p_{s} \\ 1, & \text { otherwise }\end{cases}
$$

where $p_{r}(t)$ is the cumulative charging power the node receives at time $t$, and $E_{r e}(t)$ is the residual energy at that moment.

The above equation is directly derived from the third assumption. Next, we propose our new metric.

Definition 3.2: Quality of Energy Provisioning (QoEP) of a node in the region $\Omega$ is defined as the expected portion of time in the long run that the node can sustain normal operation. Hence

$$
Q o E P=\lim _{t \rightarrow \infty} \frac{1}{t-t_{0}} \int_{t^{\prime}=t_{0}}^{t} \operatorname{IQoEP}\left(t^{\prime}\right) d t^{\prime} .
$$

We note that this concise form of QoEP not only simplifies our following analysis, but also captures the characteristics of energy provisioning performance, even when the node works in an intermittent mode.

As QoEP in Eq. (2) is a function of time, it is rather difficult to compute. We thus attempt to transform this time-dependent expression to a space-dependent one. Before that, we give the following definition.

Definition 3.3: Quality of Energy Provisioning at Location $x$ (LQoEP) of a node is defined as the expected proportion of cumulative time that the node sustains normal operation to that the node spent at $x$.

$$
\operatorname{LQoEP}(x)=\lim _{t \rightarrow \infty} \frac{\int_{t^{\prime}=t_{0}}^{t} \operatorname{IQoE} P\left(t^{\prime}\right) I\left(t^{\prime}, x\right) d t^{\prime}}{\int_{t^{\prime}=t_{0}}^{t} I\left(t^{\prime}, x\right) d t^{\prime}}
$$

where $I\left(t^{\prime}, x\right)$ is an indicator function with value 1 when a node appears at location $x$ at time $t^{\prime}$, or 0 otherwise.

With $L Q o E P(x)$, Eq. (2) can be rewritten as:

$$
\begin{aligned}
\text { QoEP } & =\lim _{t \rightarrow \infty} \frac{1}{t-t_{0}} \int_{t^{\prime}=t_{0}}^{t} \operatorname{IQoEP}\left(t^{\prime}\right) d t^{\prime} \\
& =\lim _{t \rightarrow \infty} \frac{1}{t-t_{0}} \int_{\Omega} \int_{t^{\prime}=t_{0}}^{t} \operatorname{IQoEP}\left(t^{\prime}\right) I\left(t^{\prime}, x\right) d t^{\prime} d x \\
& =\lim _{t \rightarrow \infty} \int_{\Omega} L Q o E P(x) \frac{\int_{t^{\prime}=t_{0}}^{t} I\left(t^{\prime}, x\right) d t^{\prime}}{t-t_{0}} d x \\
& =\int_{\Omega} L Q o E P(x) f_{d i s}(x) d x .
\end{aligned}
$$

By expressing the QoEP in the space domain rather than in the time domain, we are able to analyze the QoEP in a much easier way.

\subsection{Problem Description}

In this paper we prefer to investigate the QoEP of a node based on its spatial distribution, i.e., with what probability the node will stay at a specific location, rather than a specific mobility model since the analysis based on the former is more general, practical and tractable (See Section 1 in the supplemental file).

Intuitively, there may exist multiple mobility models obeying the same spatial distribution $f_{d i s}(x)$. We use $P$ to denote the set of all those qualified mobility models. As different mobility models usually lead to different values of QoEP, our task in this paper turns to pursuing upper and lower bounds of QoEP given the spatial distribution. Such theoretical results are beneficial for real applications in many aspects, such as providing 
insights into how to effectively deploy energy sources to meet requirements for energy provisioning performance. We define the lower bound of $Q o E P$ with respect to spatial distribution $f_{d i s}(x)$ as $Q o E P_{\text {min }}$, which is less than or equal to any $Q o E P$ of mobility models in $P$. We define the upper bound of $Q o E P$ by $Q o E P_{\max }$ in a similar way. Now we present our problem as follows.

Quality of Energy Provisioning Problem: Assume that there is a single source (multiple sources) and a mobile node in an $1 \mathrm{D}(2 \mathrm{D})$ region $\Omega$. The charging power at location $x \in \Omega$ is $p_{r}(x)$. Given the node's spatial distribution $f_{d i s}(x)$, maximum speed $v^{\max }$, battery capacity $E_{\pi}$ and working power $p_{s}$, the quality of energy provisioning problem is to determine $Q o E P_{\min }$ and $Q o E P_{\max }$.

\section{1D Case With Single Source}

In this section, we investigate the QoEP in 1D case with a single source. Though this case appears rather simple, it is not an easy job to derive its upper and lower bounds. Apart from its significance in theory, we emphasize that the consideration of this case also has practical meanings. For example, using a $6 \mathrm{dBi}$ receive antenna, the RF harvester designed in [28] was shown to operate at a distance of $10.4 \mathrm{~km}$ from a $1 \mathrm{MW}$ UHF television broadcast transmitter, and over $200 \mathrm{~m}$ from a cellular base transceiver station. Within such a distance, the transmitter or station serves as the primary wireless power charger, which can be modeled as $1 \mathrm{D}$ or $2 \mathrm{D}$ cases with single source (1D case may correspond to the scenario when the harvester moves on a straight road).

Suppose the source is placed at the origin, and the node's movement is confined to a line segment defined by $\left[-x_{m}, x_{m}\right]$, as shown in Fig. 2(a). Moreover, the node spatial distribution $f_{d i s}(x)$ is symmetric, namely $f_{\text {dis }}(x)=f_{\text {dis }}(-x)$ for any $x \in\left[-x_{m}, x_{m}\right]$.

\subsection{Lower Bound Analysis}

As Fig. 2 shows, the whole area is divided into two regions, $\Omega_{i}$ and $\Omega_{o}$ by $x_{T}$ and $-x_{T}$, such that a node in the region $\Omega_{i}$ is guaranteed to receive a power no less than $p_{s}$, while that in $\Omega_{o}$ is not. Hence we have $p_{r}\left(x_{T}\right)=p_{s}$. Notice that to simplify the analysis, we implicitly assume that the charging power is symmetric with respect to the origin over the line segment for this special case, i.e., $p_{r}(x)=p_{r}(-x)$. We relax this assumption for general cases as will be discussed later, namely, 1D and 2D cases with multiple sources.

Theorem 4.1: The tight lower bound of QoEP in 1D case with single source is

$$
Q o E P_{\min }=2\left(\int_{x=0}^{x_{T}} f_{d i s}(x) d x+\int_{x=x_{T}}^{x_{m}} f_{d i s}(x) \frac{p_{r}(x)}{p_{s}} d x\right) .
$$

Proof: See Section 2 in the supplemental file.

We can immediately get the following corollaries for two special cases.

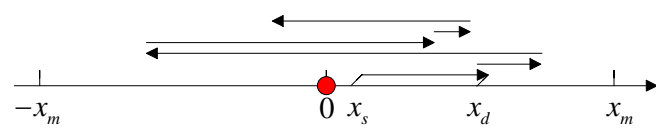

(a) Illustration of 1D Movement

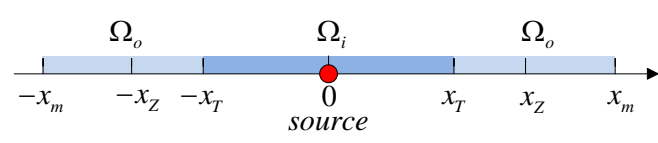

(b) Illustration of Region Partition

Fig. 2: Illustrations of 1D Case with Single Source

Corollary 4.1: For the single source case, if $p_{r}\left(x_{m}\right) \geq$ $p_{s}, Q o E P=1$.

Corollary 4.2: For the single source case, if $p_{r}(0)<p_{s}$, $Q o E P=2 \int_{x=0}^{x_{m}} \frac{p_{r}(x)}{p_{s}} f_{d i s}(x) d x$.

\subsection{Upper Bound Analysis}

In this section, we will first propose some novel conceptions to facilitate our further study, and derive a loose upper bound. Then, we investigate the tight upper bound by introducing a new analytical approach.

Due to symmetry of the spatial distribution, we only need to consider subregion $\left[0, x_{m}\right]$, and still use $\Omega_{i}$ and $\Omega_{o}$ to denote the area $\left[0, x_{T}\right]$ and $\left[x_{T}, x_{m}\right]$ respectively.

\subsubsection{Related Conceptions and Theoretical Results}

In this subsection, we will introduce some conceptions first to facilitate the following theoretical analysis in the space domain rather than in the time domain. Then we derive several useful theoretical results, which relate to loose and tight upper bounds.

First of all, we associate location $x \in \Omega_{o}$ with the expected battery energy consumption rate, i.e., the average consumption of nodal battery energy over time at $x$,

$$
\begin{aligned}
\lambda_{c}(x) & =\lim _{t \rightarrow \infty} \frac{t_{1}(x)}{t-t_{0}} \cdot 0+\lim _{t \rightarrow \infty} \frac{t(x)-t_{1}(x)}{t-t_{0}}\left(p_{s}-p_{r}(x)\right) \\
& \leq \lim _{t \rightarrow \infty} \frac{t(x)}{t-t_{0}}\left(p_{s}-p_{r}(x)\right) \quad\left(p_{s}-p_{r}(x)>0\right) \\
& =f_{\text {dis }}(x)\left(p_{s}-p_{r}(x)\right)=\lambda_{c}^{\max }(x)
\end{aligned}
$$

where $t_{1}(x)(\geq 0)$ is the cumulative time the node stays at location $x$ when its residual energy is 0 . Accordingly, the expected battery energy consumption rate $\Lambda_{c}\left(\Omega^{\prime}\right)$ for some subregion $\Omega^{\prime} \subseteq \Omega$ can be written as

$$
\Lambda_{c}\left(\Omega^{\prime}\right)=\int_{\Omega^{\prime}} \lambda_{c}(x) d x \leq \Lambda_{c}^{\max }\left(\Omega^{\prime}\right)=\int_{\Omega^{\prime}} \lambda_{c}^{\max }(x) d x .
$$

Similarly, the expected battery energy harvest rate for location $x \in \Omega_{i}$ is defined by

$$
\begin{aligned}
\lambda_{h}(x) & =\lim _{t \rightarrow \infty} \frac{t_{2}(x)}{t-t_{0}} \cdot 0+\lim _{t \rightarrow \infty} \frac{t(x)-t_{2}(x)}{t-t_{0}}\left(p_{r}(x)-p_{s}\right) \\
& \leq f_{\text {dis }}(x)\left(p_{r}(x)-p_{s}\right)=\lambda_{h}^{\max }(x)
\end{aligned}
$$

where $t_{2}(x)(\geq 0)$ is the cumulative time the node stays at location $x$ when it is fully recharged. And $\Lambda_{h}\left(\Omega^{\prime}\right)$ for some subregion $\Omega^{\prime} \subseteq \Omega_{i}$ is

$$
\Lambda_{h}\left(\Omega^{\prime}\right)=\int_{\Omega^{\prime}} \lambda_{h}(x) d x \leq \Lambda_{h}^{\max }\left(\Omega^{\prime}\right)=\int_{\Omega^{\prime}} \lambda_{h}^{\max }(x) d x .
$$


Lemma 4.1: $\Lambda_{c}\left(\Omega_{o}\right)=\Lambda_{h}\left(\Omega_{i}\right)$.

Proof: See Section 3 in the supplemental file.

Theorem 4.2: QoEP in 1D case with single source is given by

$$
Q o E P=Q o E P_{\min }+\frac{2 \Lambda_{c}\left(\Omega_{o}\right)}{p_{s}} .
$$

Proof: See Section 4 in the supplemental file.

Besides, we continue to define energy providing ability of location $x$ as the average energy a node brings into the area $\left[x, x_{m}\right]\left(x_{T} \leq x \leq x_{m}\right)$

$$
p_{e p}(x)=\lim _{t \rightarrow \infty} \frac{1}{t-t_{0}}\left(\Sigma_{m=1}^{M(t)}\left(E_{r e}^{i, m}(x)-E_{r e}^{o, m}(x)\right)\right)
$$

where $E_{r e}^{i, m}(x)$ (or $E_{r e}^{o, m}(x)$ ) is the residual energy of a node upon its $m_{t h}$ traveling across location $x$ to region $\left[x, x_{m}\right]$ (or $\left.[0, x]\right)$ during the time interval $\left[t_{0}, t\right]$, and $M(t)$ is the number of times of the node's crossing to different direction.

Denote by $\Omega^{\geq x}$ the region $\left[x, x_{m}\right]$. Based on the principle of energy conservation, the providing energy from location $x$ must be fully converted into the energy consumed in region $\Omega^{\geq x}$.

Lemma 4.2: $p_{e p}(x)=\Lambda_{c}\left(\Omega^{\geq x}\right)\left(x \in \Omega_{o}\right)$.

Further we have a related lemma as follows.

Lemma 4.3: For any location $x \in \Omega_{o}$, its energy providing ability $p_{e p}(x)$ is subject to

$$
p_{e p}(x) \leq \max \left\{0, \frac{1}{2} f_{d i s}(x) v^{\max }\left(E_{\pi}-\int_{y=x_{T}}^{x} \frac{\left(p_{s}-p_{r}(y)\right)}{v^{\max }} d y\right)\right\} .
$$

Proof: See Section 5 in the supplemental file.

For convenience, we refer to the maximum value of $p_{e p}(x)$ as $\tilde{p}_{e p}^{\max }(x)$. The proof of Lemma 4.3 reveals that the faster a node moves, the larger $p_{e p}(x)$ it may gain. In essence, the faster speed accelerates the energy exchange between the region $\Omega_{i}$ and $\Omega_{o}$, and finally leads to an improvement of QoEP.

Next, we present a lemma as follows.

Lemma 4.4: For any $x \in \Omega_{o}\left(x_{T} \leq x<x_{m}\right)$,

$$
\Lambda_{c}\left(\Omega_{o}\right)=\Lambda_{c}\left(\Omega_{o}-\Omega^{\geq x}\right)+p_{e p}(x)
$$

and

$$
\Lambda_{c}\left(\Omega_{o}\right) \leq \Lambda_{c}^{\max }\left(\Omega_{o}-\Omega^{\geq x}\right)+\tilde{p}_{e p}^{\max }(x)
$$

where

$$
\tilde{p}_{e p}^{\max }(x)=\max \left\{0, \frac{1}{2} f_{d i s}(x) v^{\max }\left(E_{\pi}-2 \int_{y=x_{T}}^{x} \frac{\left(p_{s}-p_{r}(y)\right)}{v^{\max }} d y\right)\right\} .
$$

Proof: See Section 6 in the supplemental file.

\subsubsection{Loose Upper Bound}

Based on the proposed conceptions and theoretical results, we can derive a loose upper bound of QoEP for $1 \mathrm{D}$ case with single source.

Let $x=x_{T}$ in Lemma 4.2 and Lemma 4.3, and combine them with Theorem 4.1 and Theorem 4.2, we have the following theorem.

Theorem 4.3: The loose upper bound of QoEP in 1D case with single source is

$$
Q o E P \leq Q o E P_{\min }+\frac{v^{\max } E_{\pi}}{p_{s}} f_{d i s}\left(x_{T}\right) .
$$

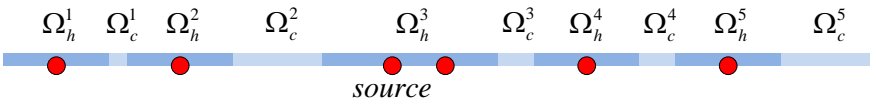

Fig. 3: Illustration of 1D Case with Multiple Sources

\subsubsection{Tight Upper Bound}

In this subsection, we intend to derive the tight upper bound of QoEP in 1D case with single source. It is an extremely challenging task since we have to find the optimal mobility model that achieves the upper bound of QoEP in order to prove the tightness of the bound. Towards this goal, we present a novel analytical approach - flow pattern analysis to facilitate our analysis. Due to space limit, we only present the main result of the tight upper bound as follows, and refer the reader to Section 7 in the supplemental file for details.

Theorem 4.4: The tight upper bound of QoEP in 1D case with single source is

$$
Q o E P_{\max }=Q o E P_{\min }+2 \frac{\min \left\{\Lambda_{c}^{o p t}\left(\Omega_{o}\right), \Lambda_{h}^{o p t}\left(\Omega_{i}\right)\right\}}{p_{s}} .
$$

where $\Lambda_{c}^{o p t}\left(\Omega_{o}\right)$ and $\Lambda_{h}^{o p t}\left(\Omega_{i}\right)$ are the maximum expected battery energy consumption rate for region $\Omega_{o}$ and the maximum expected battery energy harvest rate for the region $\Omega_{i}$, respectively.

\section{1D Case With Multiple Sources}

In this section, we attempt to extend the results to multiple sources case.

For this case, we assume that there is a 1D network consisting of $N$ sources, as illustrated in Fig. 3. The whole area is partitioned into multiple regions, which can be classified into two types according to whether nodes within the regions can receive a power no less than $p_{s}$ or not, as depicted in dark color and light color blocks respectively. We denote them as $\Omega_{h}^{p}(p=$ $1,2,3, \ldots, P)$ and $\Omega_{c}^{q}(q=1,2,3, \ldots, Q)$ respectively. Similar to Theorem 4.1, we have the lower bound as follows.

Theorem 5.1: The tight lower bound of QoEP in 1D case with multiple sources is

$$
\text { QoEP } P_{\text {min }}=\sum_{p=1}^{P} \int_{\Omega_{h}^{p}} f_{\text {dis }}(x) d x+\sum_{q=1}^{Q} \int_{\Omega_{c}^{q}} f_{d i s}(x) \frac{p_{r}(x)}{p_{s}} d x .
$$

Proof: Since the proof is similar to Theorem 4.1, we omit it here.

Nevertheless, the calculation of upper bound in this case turns to be much more complicated. For example, suppose that the optimal expected battery energy consumption rate for region $\Omega_{c}^{2}$ in Fig. 3 is $\Lambda_{c}^{o p t+}\left(\Omega_{c}^{2}\right)$ in its left side and $\Lambda_{c}^{\text {opt }}\left(\Omega_{c}^{2}\right)$ in its right side, which are obtained by launching main flows from the left end point and the right end point into $\Omega_{c}^{2}$. Then we have the following theorem.

Theorem 5.2: The loose upper bound of QoEP in 1D case with multiple sources is

$$
Q o E P \leq Q o E P_{\min }+\min \left\{\sum_{p=1}^{P} \Lambda_{h}^{o p t}\left(\Omega_{h}^{p}\right)+\sum_{q=1}^{Q} \Lambda_{c}^{o p t}\left(\Omega_{c}^{q}\right)\right\}
$$


where $\Lambda_{h}^{\text {opt }}\left(\Omega_{h}^{p}\right)=\min \left\{\Lambda_{h}^{\text {opt+ }}\left(\Omega_{h}^{p}\right)+\Lambda_{h}^{\text {opt- }}\left(\Omega_{h}^{p}\right), \Lambda_{h}^{\max }\right.$ $\left.\left(\Omega_{h}^{p}\right)\right\}$ and $\Lambda_{c}^{o p t}\left(\Omega_{c}^{q}\right)=\min \left\{\Lambda_{c}^{o p t+}\left(\Omega_{c}^{q}\right)+\Lambda_{c}^{o p t-}\left(\Omega_{c}^{q}\right)\right.$, $\left.\Lambda_{c}^{\max }\left(\Omega_{c}^{q}\right)\right\}$.

Proof: The spirit of the proof is similar to Theorem 4.4, we omit it here.

\section{2D Case With Multiple Sources}

In this section, we are dedicated to analyze the upper and lower bounds of QoEP in general 2D case with multiple sources.

For the lower bound of QoEP, we have the following theorem.

Theorem 6.1: The tight lower bound of QoEP in 2D case with multiple sources is

$$
Q o E P_{\min }=\int_{\Omega} f_{\text {dis }}(x, y) J\left(\frac{p_{r}(x, y)}{p_{s}}\right) d x d y
$$

where $J(x)=x$ for $0 \leq x \leq 1$ and 1 for $x>1$.

Proof: See Section 8 in the supplemental file.

Similarly, for the upper bound of QoEP, we have

Theorem 6.2: The loose upper bound of QoEP in 2D case with multiple sources is

$$
Q o E P \leq Q o E P_{\text {min }}+\frac{v^{m a x} E_{\pi}}{2 p_{s}} \sum_{\ell \in L} \int_{\ell} f_{d i s}(x, y) d s
$$

where $L$ is the set of curves in the 2D plane with each point $(x, y)$ on it satisfying $p_{r}(x, y)=p_{s}$.

Proof: See Section 9 in the supplemental file.

\section{Discussion}

From Theorem 4.3 and Theorem 6.2, we observe that node speed and battery capacity have the same impacts on the upper bounds of QoEP in both 1D and 2D cases. Actually, we have a more general finding as the following theorem discloses.

Theorem 7.1: Node speed $v^{\max }$ and battery capacity $E_{\pi}$ have the same influence on QoEP.

Proof: See Section 10 in the supplemental file.

In addition, we can easily extend the results for 1D and $2 \mathrm{D}$ cases to the 3D case. By using the similar analysis for the 2D case with multiple sources, we can derive the tight lower bound and the loose upper bound for the 3D case. In particular, for the tight lower bound, we need to take the integral of the whole 3D space similar to Eq. (16). Further, for the loose tight bound, compared with Eq. (17), the second term is indeed the integration of all surfaces with each point $(x, y, z)$ on it satisfying $p_{r}(x, y, z)=p_{s}$.

\section{Simulation Results}

In this section, we present simulation results to verify our findings. Throughout the simulations, we adopt the empirical charging model proposed by [29]. In this model, the received power $p_{r}(d)$ by a node can be quantified by $p_{r}(d)=\frac{\alpha}{(d+\beta)^{2}}$, where $d$ is the distance from the source to the node, and $\alpha$ and $\beta$ are known constants. Table 2 lists the default simulation parameters.
TABLE 2: Simulation Parameters

\begin{tabular}{|c|l|}
\hline Parameter & Value \\
\hline \hline$\alpha\left(W \cdot m^{2}\right)$ & $4.328 \times 10^{-4}$ \\
\hline$\beta(m)$ & 0.2316 \\
\hline$p_{s}(W)$ & $1 \times 10^{-3}$ \\
\hline ratio $\left(=v^{\text {max }} / v^{\text {min }}\right)$ & $1,2,10,100$ \\
\hline
\end{tabular}

\subsection{D Case with Single Source}

We consider two mobility models for this case.

\subsubsection{Random Waypoint Mobility Model}

The spatial distribution of the Random Waypoint Mobility Model (RWMM) in 1D case is given by $f_{\text {dis }}(x)=$ $-\frac{3}{4 x_{m}^{3}} x^{2}+\frac{3}{4 x_{m}}$ for $-x_{m} \leq x \leq x_{m}$ [10], as is illustrated in Fig. 4(a). Comparison of the simulation data points on the dotted curve with the theoretical data points on the solid curve shows they are in good agreement, when we set $x_{m}=1$ and randomly choose speed between [0.01,1].

As shown in Fig. 4(b), when $E_{\pi}$ decreases, the simulation results of QoEP approach to the lower bound 0.8519 . The ratio in Fig. 4(b) refers to $v^{\max } / v^{\min }$ while $v^{\max }$ is kept constant and equals 0.1 . Note that the speed of a node for each movement is randomly selected from $\left[v^{\min }, v^{\max }\right]$, then different ratios actually lead to different instances of RWMM. We observe that with different ratios, QoEPs are always bounded by the upper and lower bounds (note that QoEP is obtained by cutting the trajectory of the node into tiny pieces and regarding the charging power as constant for each piece). Specifically, for ratio=1, 2, 10 and 100, the difference between their QoEPs and the upper bound rises from $0.19 \%$ to $4.47 \%$ near $10^{-3}$, and from $0.01 \%$ to $7.29 \%$ at $10^{-2}$. Moreover, the RWMM of ratio=1, which implies that the node moves with an invariable speed $v^{\max }$, yields a QoEP very close to the upper bound.

Likewise, the QoEPs increase monotonically with $v^{\max }$ when $E_{\pi}=0.001$ as Fig. 4(c) shows. In addition, the observation that the influence of maximum speed on QoEP is the same as that of battery capacity corroborates Theorem 7.1.

\subsubsection{Random Multi-State Mobility Model}

We proceed to evaluate our findings in a more complicated case. Suppose that a node moves within the region $[-0.9,0.9]$ following a so-called Random MultiState Mobility Model (RMSMM). In particular, it turns to the positive direction of the $x$ axis with probability $p(x)=1 \quad($ or $\quad 0.2,0.8,0.2,0.8,0.2,0.8,0.2,0.8,0)$ when it reaches location $x=-0.9$ (or $-0.7,-0.5,-0.3,-0.1,0.1,0.3,0.5,0.7,0.9)$, or turns to the opposite direction with the remaining probability. Meanwhile, the node changes its speed to a value randomly selected within the range of $\left[v^{\min }, v^{\max }\right]$. At the other locations, the node moves with a constant direction and speed.

Apparently, the node's transition between spatial regions can be cast as a Markov chain with nine subregions regarded as nine states. The spatial distribution sketched 


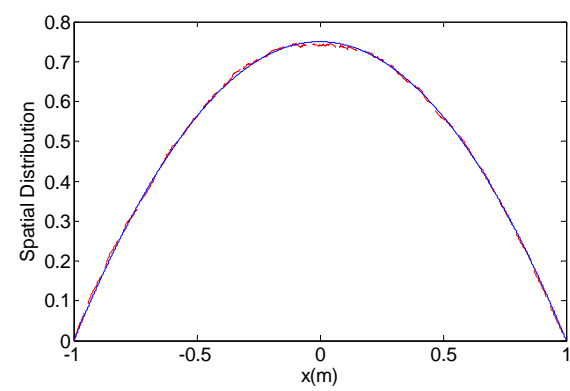

(a) Spatial Distribution of RWMM

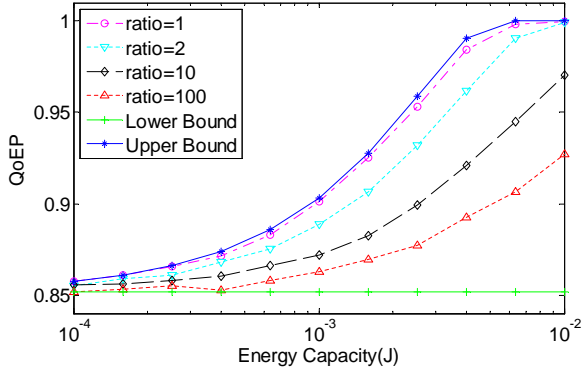

(b) QoEP Vs. Energy Capacity $\left(v^{\max }=0.1\right)$

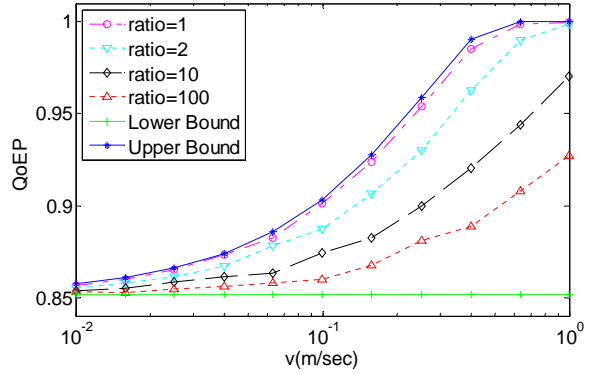

(c) QoEP Vs. Maximum Speed $\left(E_{\pi}=0.001\right)$

Fig. 4: Simulation Results of Random Waypoint Mobility Model in Single Source Case

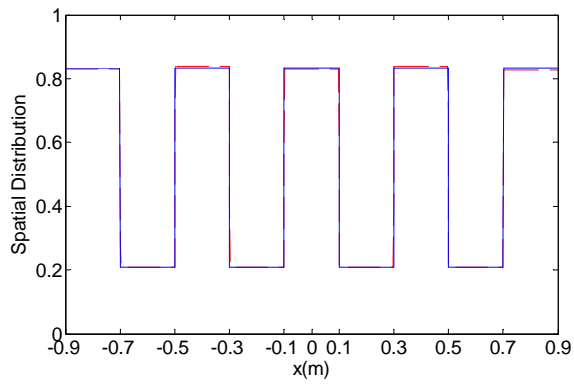

(a) Spatial Distribution of RMSMM

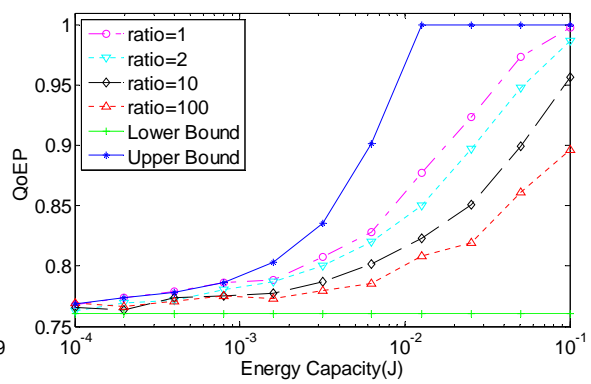

(b) QoEP Vs. Energy Capacity $\left(v^{\max }=0.1\right)$

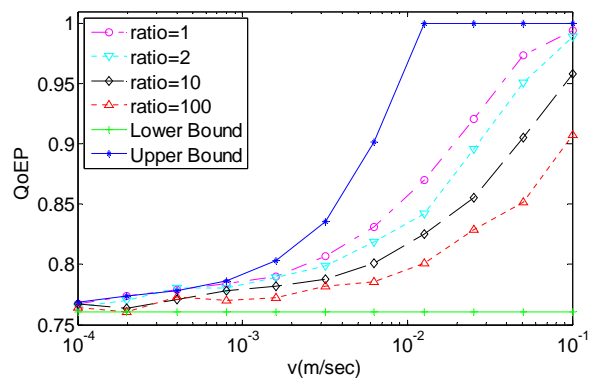

(c) QoEP Vs. Maximum Speed $\left(E_{\pi}=0.1\right)$

Fig. 5: Simulation Results of Random Multi-State Mobility Model in Single Source Case

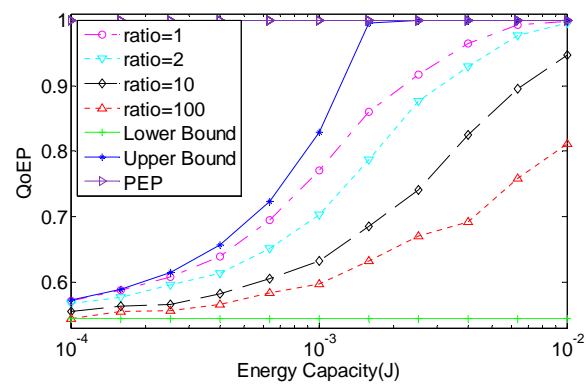

(a) QoEP Vs. Energy Capacity $\left(v^{\max }=1\right)$

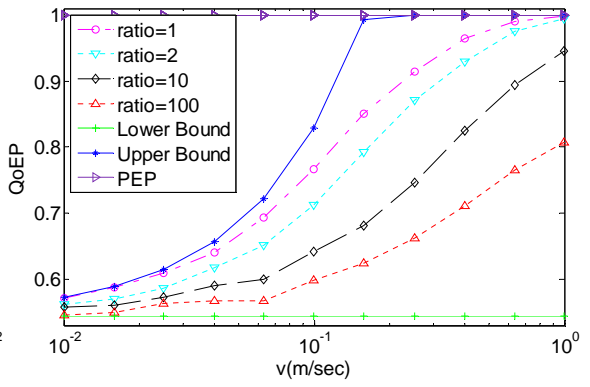

(b) QoEP Vs. Maximum Speed $\left(E_{\pi}=0.01\right)$

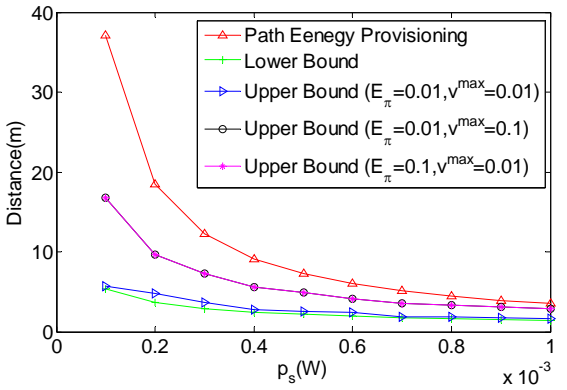

Fig. 7: Illustration of Distance Comparison for Triangular Placement
Fig. 6: Simulati
Sources Case

in Fig. 5(a) resembles a square wave with minimum and maximum value 0.2083 and 0.8333 . The QoEPs with varying battery capacity and speed are demonstrated in Fig. 5(b) and Fig. 5(c) respectively, which departure from upper bound a larger distance compared with that in RWMM. In Fig. 5(b), for example, when the energy capacity is about $10^{-2}$, the difference between QoEP and the upper bound increases from $12.30 \%$ to $19.20 \%$ when the ratio increases from 1 to 100 . This is because the higher tendency of the node to move locally reduces the opportunity of sufficient energy exchange.

\subsection{D Case with Multiple Sources}

For 1D case with multiple sources, we are concerned with the Random Walk Mobility Model when sources are equidistantly distributed with distance interval $d$. Due to uniform spatial distribution of this mobility model and symmetry of the sources, we can consider only a subregion $[-d / 2, d / 2]$ with a reference source placed at the origin. Accordingly, the mobility model we concerned is converted into the Random Walk with Reflection Mobility Model (RWRMM) proposed in [30]. For this mobility model, each movement of a node occurs in a constant distance traveled $l=0.4 d$, at the end of which a new direction and speed are calculated. If the node reaches a boundary, it "bounces" off the border and continues along this new direction. It is obvious that RWRMM also follows uniform distribution.

To evaluate the performance of our work, we compare it with that presented in [9]. Specifically, we adapt the approach in [9] to 1D case, by applying the following equation to determine the distance $d$ between adjacent sources: $\frac{1}{d} \int_{r=0}^{d}\left[\frac{\alpha}{(r+\beta)^{2}}+\frac{\alpha}{(d-r+\beta)^{2}}\right] d r=p_{s}$. Solving this equation we obtain $d=\frac{2 \alpha}{\beta p_{s}}-\beta$. Indeed, this is exactly the maximum distance to guarantee path energy provisioning, whose QoEP equals 1 as [9] implies.

In Fig. 6(a) and 6(b), we plot the QoEP to varying energy capacity and maximum speed. It shows that the 
QoEP always equals 1 for path energy provisioning, which is referred to as PEP in these figures, as we set $d=\frac{2 \alpha}{\beta p_{s}}-\beta=3.5059$. In contrast, the QoEPs of real cases, and even that of upper bound are much smaller, especially with a relatively low energy capacity or maximum speed.

We proceed to evaluate the gap between our solution and path energy provisioning. We vary the nodal working power $p_{s}$ and compute the maximum distance between adjacent sources in triangular placement to guarantee the QoEP equals 1 according to the upper and lower bounds as well as that for path energy provisioning [9]. It can be seen from Fig. 7 that the distance for upper bound increases as $E_{\pi}$ or $v^{\max }$ increases, and $E_{\pi}$ and $v^{\max }$ have the same impact on distance. We conclude that our proposed upper and lower bounds are more practical than path energy provisioning as it takes into consideration the constraints of node speed and energy capacity. The results of upper bound and lower bound can be used to better estimate the distance between sources for deployment.

\subsection{D Case with Multiple Sources}

For 2D case with multiple sources, we are concerned with the Random Direction Mobility Model (RDMM) [31].

Suppose there are four sources $\left\{s_{1}, s_{2}, s_{3}, s_{4}\right\}$ deployed on a $4 m * 4 m$ region with coordinates $(1,2),(1.5,2.6)$, $(3,2.8)$ and $(3.5,0.2)$, respectively. Moreover, a node obeying RDMM travels to the border of the region at constant speed, and then randomly chooses another angular direction and continues the process. This simple mobility model is proven to yield a uniform spatial distribution. We thus compute the upper and lower bounds using this result.

Fig. 8 depicts the relation between the simulation results of QoEP and the upper and lower bounds with respect to battery energy capacity and node speed. Again in the figure the upper and lower bounds strictly hold, and battery capacity and node speed have the same impact on QoEP.

\section{Conclusion}

In this paper, we have studied the quality of energy provisioning for wireless power transfer technology. Our work mainly focuses on analyzing upper and lower bounds of QoEP in 1D and 2D cases. The theoretical results show that the lower bounds in both cases have nothing to do with node speed and battery capacity. In contrast, both of the factors affect the upper bounds to the same extent.

In future work, we will attempt to extend the flow pattern analysis approach to general 1D and 2D cases, and thereby pursue the tight upper bounds accordingly.

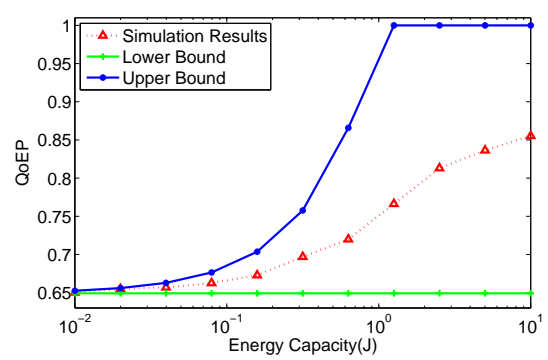

(a) QoEP Vs. Energy Capacity $\left(v^{\max }=0.001\right)$

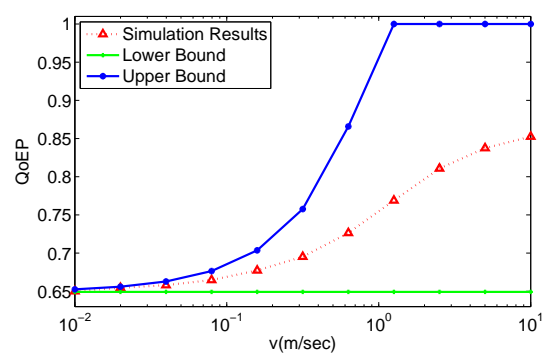

(b) QoEP Vs. Speed $\left(E_{\pi}=0.001\right)$

Fig. 8: Simulation Results of Random Direction Mobility Model in 2D Case

\section{ACKNOWLEDGMENT}

This work is supported by National 973 project of China under Grants No.2012CB316200, No.2012CB316201, No.2014CB340300 and No.2014CB340303, National Natural Science Foundation of China under Grants No.61133006, No.61373130, No.61321491 and No.61272443, National Key Project of China under Grant No.2013ZX01033002-003, Research and Innovation Project for College Graduate Students of Jiangsu Province in 2012 under Grant No.CXZZ12_0056, the Shanghai Science and Technology fund 12PJ1404900 and JiangsuSF under Grant No.BK2011051.

\section{REFERENCES}

[1] A. Kurs, A. Karalis, R. Moffatt, J. D. Joannopoulos, P. Fisher, and M. Soljačić, "Wireless power transfer via strongly coupled magnetic resonances," science, vol. 317, no. 5834, pp. 83-86, 2007.

[2] "www.powercastco.com."

[3] "http://www.seattle.intel-research.net/wisp/."

[4] "http://www.laptopmag.com/reviews/laptops/dell-latitude3330.aspx."

[5] "www.powermat.com."

[6] "http:/ / evworld.com/news.cfm?newsid=24420."

[7] "http://www.friendsofcrc.ca/projects/sharp/sharp.html."

[8] "http://equity.co.kr/upfile/issue/2012/05/10/1336611859340.pdf."

[9] S. He, J. Chen, F. Jiang, D. K. Y. Yau, G. Xing, and Y. Sun, "Energy provisioning in wireless rechargeable sensor networks," in INFOCOM, 2011.

[10] C. Bettstetter and C. Wagner, "The spatial node distribution of the random waypoint model," in WMAN, 2002.

[11] T.-C. Chiu, Y.-Y. Shih, A.-C. Pang, J.-Y. Jeng, and P.-C. Hsiu, "Mobility-aware charger deployment for wireless rechargeable sensor networks." in APNOMS, 2012.

[12] H. Dai, L. Xu, X. Wu, C. Dong, and G. Chen, "Impact of mobility on energy provisioning in wireless rechargeable sensor networks," in WCNC, 2013.

[13] L. Xie, Y. Shi, Y. T. Hou, and W. Lou, "Wireless power transfer and applications to sensor networks," IEEE Wireless Communications Magazine.

[14] M. Buettner, R. Prasad, M. Philipose, and D. Wetherall, "Recognizing daily activities with RFID-based sensors," in Ubicomp, 2009. 
[15] C. Greene, D. Harrish, D. Kalp, and W. Tauche, "Making wireless sensor networks truly wireless using rf power."

[16] R. P. Wicaksono, G. K. Tran, K. Sakaguchi, and K. Araki, “Wireless Grid: Enabling Ubiquitous Sensor Networks with Wireless Energy Supply," pp. 1-5, 2011.

[17] H. Dai, Y. Liu, G. Chen, X. Wu, and T. He, "Safe charging for wireless power transfer," in INFOCOM, 2014.

[18] B. Tong, Z. Li, G. Wang, and W. Zhang, "How wireless power charging technology affects sensor network deployment and routing," in ICDCS, 2010.

[19] Z. Li, Y. Peng, W. Zhang, and D. Qiao, "J-RoC: a joint routing and charging scheme to prolong sensor network lifetime," in IPSN, 2011.

[20] Y. Shi, L. Xie, Y. T. Hou, and H. D. Sherali, "On renewable sensor networks with wireless energy transfer," in INFOCOM, 2011.

[21] L. Xie, Y. Shi, Y. T. Hou, W. Lou, and H. D. Sherali, "On traveling path and related problems for a mobile station in a rechargeable sensor network," in MobiHoc. ACM, 2013.

[22] M. Zhao, J. Li, and Y. Yang, "Joint mobile energy replenishment and data gathering in wireless rechargeable sensor networks," in ITC, 2011.

[23] S. Guo, C. Wang, and Y. Yang, "Mobile data gathering with wireless energy replenishment in rechargeable sensor networks," in INFOCOM, 2013.

[24] L. Xie, Y. Shi, Y. T. Hou, W. Lou, H. D. Sherali, and S. F. Midkiff, "Bundling mobile base station and wireless energy transfer: Modeling and optimization," in INFOCOM, 2013.

[25] H. Dai, L. Jiang, X. Wu, D. K. Yau, G. Chen, and S. Tang, "Near optimal charging and scheduling scheme for stochastic event capture with rechargeable sensors," in MASS, 2013.

[26] S. Zhang, J. Wu, and S. Lu, "Collaborative mobile charging for sensor networks," in MASS, 2012.

[27] H. Dai, X. Wu, L. Xu, G. Chen, and S. Lin, "Using minimum mobile chargers to keep large-scale wireless rechargeable sensor networks running forever." ICCCN, 2013.

[28] A. N. Parks, A. P. Sample, Y. Zhao, and J. R. Smith, "A wireless sensing platform utilizing ambient RF energy," in RWS, 2013.

[29] L. Fu, P. Cheng, Y. Gu, J. Chen, and T. He, "Minimizing charging delay in wireless rechargeable sensor networks." INFOCOM, 2013.

[30] J.-Y. L. Boudec and M. Vojnovic, "Perfect simulation and stationarity of a class of mobility models," in INFOCOM, 2005.

[31] E. M. Royer, P. M. Melliar-Smith, and L. E. Moser, "An analysis of the optimum node density for ad hoc mobile networks," in ICC, 2001.

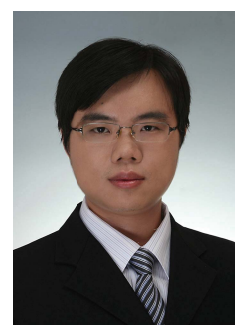

Haipeng Dai received the B.S. degree in the Department of Electronic Engineering from Shanghai Jiao Tong University, Shanghai, China, in 2010. He is currently pursuing the Ph.D. degree at the Department of Computer Science and Technology in Nanjing University, Nanjing, China. His research interests are mainly in the areas of wireless sensor networks and wireless power transfer.

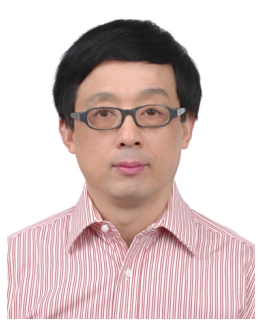

Guihai Chen received B.S. degree in computer software from Nanjing University in 1984, M.E. degree in computer applications from Southeast University in 1987, and Ph.D. degree in computer science from the University of Hong Kong in 1997. He is a professor and deputy chair of the Department of Computer Science, Nanjing University, China. He had been invited as a visiting professor by many foreign universities including Kyushu Institute of Technology, Japan in 1998, University of Queensland, Australia in 2000, and Wayne State University, USA during Sept. 2001 to Aug. 2003. He has a wide range of research interests with focus on sensor networks, peer-to-peer computing, high-performance computer architecture and combinatorics.

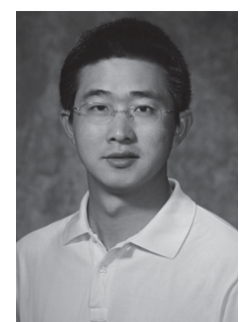

Chonggang Wang (SM'09) received the $\mathrm{PhD}$ degree from Beijing University of Posts and Telecommunications (BUPT), China in 2002. $\mathrm{He}$ is currently a Senior Research Staff with InterDigital Communications with focuses on Machine-to-Machine (M2M) communications and Internet of Things (loT) R\&D activities including technology development and standardization. Before joining InterDigital in 2009, he had conducted various researches with NEC Laboratories America, AT\&T Labs Research, University of Arkansas, and Hong Kong University of Science and Technology. He (co-)authored more than 100 journal/conference articles and book chapters. He is on the editorial board for several journals including IEEE Communications Magazine and IEEE Transactions on Network and Service Management. He was/is co-organizing several special issues respectively for IEEE Network magazine, IEEE Communications magazine, IEEE Communications Surveys and Tutorials, etc. He received Outstanding Leadership Award from IEEE GLOBECOM 2010 and InterDigitals 2012 Innovation Award. He is the vice-chair of IEEE ComSoc Multimedia Technical Committee (MMTC).

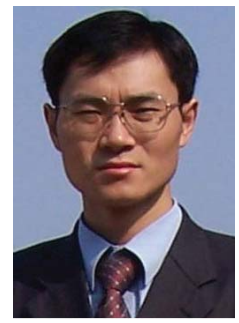

Shaowei Wang (S'06-M'07-SM'13) received the $\mathrm{BS}, \mathrm{MS}$ and $\mathrm{PhD}$ degrees in electronic engineering from Wuhan University, China, in 1997, 2003 and 2006, respectively (with honors). From 1997 to 2001, he was a research scientist of China Telecom, China. He joined the School of Electronic Science and Engineering of Nanjing University, China, in 2006, where he has been an Associate Professor since 2008. From 2009 to 2011, He was also with LAMDA group, Nanjing University, China. From 2012 to 2013, he is/was also with Stanford University, CA, and The University of British Columbia, Canada. His research interests are mainly in wireless communications and networks, optimization and machine learning. Currently, his research focuses on resource allocation in wireless networks, cognitive radio, cellular networks planning, and traffic engineering in telecommunications. In these areas he has published more than 50 papers in leading journals and conference proceedings. He is the guest editor of IEEE Wireless Communications special issue on "Enhancing Spectral Efficiency for LTE-Advanced and Beyond Cellular Networks". He serves/ed as Publicity Co-Chair of the IEEE ISCC12/13, Tutorial CoChair of the IEEE SmartGridComm12, Symposium Co-Chair of the IEEE ICNC14.

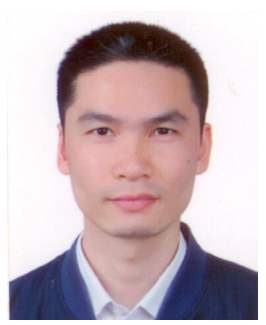

Xiaobing Wu received Ph.D. degree in computer science from Nanjing University (NJU) in 2009 where he is an Assistant Professor. He had been with China Merchants Bank for two years before moving to NJU in 2005. He visited the Illinois Institute of Technology as a research scholar from Oct. 2007 to Oct. 2008. His research interests are mainly in the areas of wireless networking. He is a member of the IEEE and the ACM.

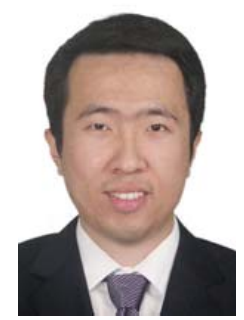

Fan $\mathbf{W u}$ is an associate professor in the Department of Computer Science and Engineering at Shanghai Jiao Tong University, P. R. China. $\mathrm{He}$ received his B.S. in Computer Science from Nanjing University in 2004, and Ph.D. in Computer Science and Engineering from the State University of New York at Buffalo in 2009. He has visited the University of Illinois at UrbanaChampaign (UIUC) as a Post Doc Research Associate. His current research interests include wireless networking, algorithmic mechanism design, and privacy preservation. He received Excellent Young Scholar award of Shanghai Jiao Tong University in 2011, and Pujiang Scholar award in 2012. He is a member of ACM, CCF, and IEEE. For more information, please visit http://www.cs.sjtu.edu.cn/ fwu/. 